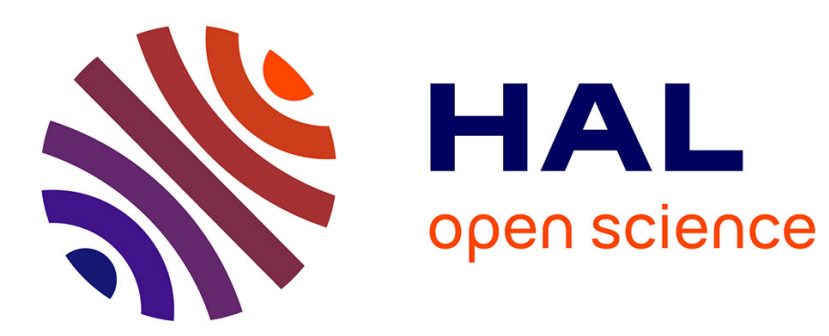

\title{
A Bayesian approach for the separation of the acoustic and the correlated aerodynamic wall pressure fluctuations
}

Alice Dinsenmeyer, Quentin Leclere, Jérôme Antoni, Emmanuel Julliard

\section{- To cite this version:}

Alice Dinsenmeyer, Quentin Leclere, Jérôme Antoni, Emmanuel Julliard. A Bayesian approach for the separation of the acoustic and the correlated aerodynamic wall pressure fluctuations. Journal of the Acoustical Society of America, 2021, 149 (6), pp.4410-4421. 10.1121/10.0005243 . hal-03268486v2

\section{HAL Id: hal-03268486 \\ https://hal.science/hal-03268486v2}

Submitted on 12 Jul 2021

HAL is a multi-disciplinary open access archive for the deposit and dissemination of scientific research documents, whether they are published or not. The documents may come from teaching and research institutions in France or abroad, or from public or private research centers.
L'archive ouverte pluridisciplinaire HAL, est destinée au dépôt et à la diffusion de documents scientifiques de niveau recherche, publiés ou non, émanant des établissements d'enseignement et de recherche français ou étrangers, des laboratoires publics ou privés. 


\title{
A Bayesian approach for the separation of the acoustic and the correlated aerodynamic wall pressure fluctuations
}

\author{
Alice Dinsenmeyer ${ }^{1}$, Quentin Leclère ${ }^{1}$, Jérôme Antoni ${ }^{1}$, and Emmanuel Julliard ${ }^{2}$ \\ ${ }^{1}$ Univ Lyon, INSA Lyon, LVA, EA677, 69621 Villeurbanne, France \\ ${ }^{2}$ Airbus Operations S.A.S, Acoustics Department, Toulouse, France
}

2021, June

\begin{abstract}
When performing measurements with wall-installed microphone array, the turbulent boundary layer (TBL) that develops over the measuring system can induce pressure fluctuations that are much greater than those of acoustic sources. It then becomes necessary to process the data to extract each component of the measured field. For this purpose, it is proposed in this paper to decompose the measured spectral matrix into the sum of matrices associated with the acoustic and aerodynamic contributions. This decomposition exploits the statistical properties of each pressure field. On the one hand, assuming that the acoustic contribution is highly correlated over the sensors, the rank of the corresponding cross-spectral matrix is limited to a finite number. On the other hand, the correlation structure of the aerodynamic noise matrix is constrained to resemble a Corcos-like model, with physical parameters estimated within the separation procedure. This separation problem is solved by a Bayesian inference approach, which takes into account the uncertainties on each component of the model. The performance of the method is first evaluated on wind tunnel measurements and then on a particularly noisy industrial measurement setup: microphones flush mounted on the fuselage of a large aircraft.
\end{abstract}

\section{Introduction}

Multichannel measurements are often performed in order to understand the noise emissions. The use of wall-installed or flush-mounted microphones is often required for the measurements performed in the presence of a flow, which can be outdoor, in underwater environment, in wind-tunnel, on moving vehicles, etc. In this case, a Turbulent Boundary Layer (TBL) develops on the measurement wall and the measured pressure field results from two types of pressure fluctuations: those induced by the TBL, called the aerodynamic contribution, and those induced by the acoustic sources, if any, called the acoustic contribution. The two contributions need to be investigated separately in order to achieve these two objectives.

A first objective is to study the acoustic or aeroacoustic sources, through quantification and localization provided by image post-processing. In this case, the aerodynamic contribution needs to be mitigated so as not to contaminate the analysis of the acoustic field.

The second objective of wall-pressure measurements is the study of some features of the flow. This is required for the prediction of the vibro-acoustic excitations, which is a challenge for many applications such as the control of the fatigue loading on structures, the reduction of the vehicle interior noise or the noise generated by piping systems with internal turbulent flow.
These studies require an acoustic decontamination of the measurements.

For these two purposes, several strategies have been developed to perform the separation of the acoustic and TBL pressure fluctuations in the measurement data. The existing post-processing approaches make use of the different correlation properties of the two fields. Indeed, the acoustic field is highly correlated over a classical microphone array, whereas the TBL field has a much smaller correlation, with a structure that has been described by several experimental models [1]. In the wavenumber plane, this difference in correlation lengths translates into two different wavenumber areas, and the wavenumber filtering method [2] exploits the fact that the two contributions of interest span different wavenumber domains to perform the separation through an integration over the wavenumber area of each contribution. However, this approach requires a large spatial sampling and thus a high number of sensors, as well as a clear distinction between the two wavenumber areas, which is not always the case at low frequencies or high convection speeds, or in presence of aliasing effects.

During the last decade, several other methods have been proposed, based on the assumption that the TBL field is uncorrelated over the microphone (see Ref. 3 for an overview of these methods). This assumption is sufficient at high frequencies and for the use of arrays 
with large microphone interspacing, but these methods show limited performance at low frequencies where this assumption no longer holds true.

In the present paper, an extension of the Probabilistic Factor Analysis (PFA) approach introduced in a previous work [3] is proposed, in order to account for the correlation of the TBL field and significantly improve the separation results at low frequencies. The proposed separation approach does not make use of any noise-free reference measurement and of any assumption about the acoustic source localization or propagation. In the following, this separation approach is called Probabilistic Factor Analysis with Correlated noise model, written PFA-Corr.

In this paper, the separation is performed through a fitting of the measured data over a structured covariance model, which is first described in Sec. 2. In Sec. 3, it is explained how this model accounts for the uncertainty about the acoustic and aerodynamic contributions thanks to the use of a Bayesian inference framework. Especially, the TBL parameters - the convection speed and coherence lengths - are estimated within the separation procedure in order to improve the precision of the separation.

In Sec. 4, the separation approach is validated on wind-tunnel measurements and some applications on inflight measurement are finally given in Sec. 5 .

\section{Probabilistic modeling of the problem}

\subsection{Problem statement}

The problem addressed in this paper is the separation of the contributions coming from acoustic sources and from the TBL pressure fluctuations measured simultaneously by an array of $M$ microphones. At one frequency, the Fourier coefficients of the pressure measured by the sensors can be arranged in a vector $\boldsymbol{y}$ of $M$ complex values. This pressure vector can thus be written as the sum of an acoustic contribution $\boldsymbol{a}$, an aerodynamic contribution $\boldsymbol{n}$ and noise term $\boldsymbol{\epsilon}$ containing all the additive sources of noise and errors:

$$
\boldsymbol{y}=\boldsymbol{a}+\boldsymbol{n}+\boldsymbol{\epsilon}
$$

When dealing with pressure fields which are statistically stationary in time, it is common to store the measurements in the form of a Cross-Spectral Matrix (CSM), which is defined by the covariance matrix of the measured Fourier coefficients. This is estimated by averaging over $N_{s}$ successive overlapping windowed time signal segments (hereafter called snapshots), following
Welch's periodogram method:

$$
\hat{\boldsymbol{S}}_{y y}=\frac{1}{N_{s}} \sum_{j=1}^{N_{s}} \boldsymbol{y}_{j} \boldsymbol{y}_{j}^{H}, \quad j=1, \ldots, N_{s},
$$

where $\hat{\imath}$ is used to indicate an estimated quantity and where the superscript $H$ indicates the complex conjugate transpose (or Hermitian) operator. Then, replacing $\boldsymbol{y}$ in Eq. (2) by the sum of Eq. (1), the theoretical CSM - i.e. assuming an infinite number of snapshots, such that the cross-terms tend to zero - of the measurements reads

$$
\boldsymbol{S}_{y y}=\boldsymbol{S}_{a a}+\boldsymbol{S}_{n n}+\boldsymbol{S}_{\epsilon \epsilon} .
$$

The objective of the separation problem is to estimate each matrix in the above equation (3) from the measurements. In order to make the inverse problem identifiable, the proposed approach makes use of some model for the correlation structure of each quantity. To benefit from an intuitive regularization, the problem is solved within a Bayesian framework. It allows to take advantage from the prior information about this problem, which can be statistical, physical, coming from experimental or numerical knowledge.

One solution of this fitting problem is given by the set of unknowns that has the highest joint probability knowing the measurement data, which is the posterior probability. This reads

$$
\left[\boldsymbol{S}_{a a}^{\star}, \boldsymbol{S}_{n n}^{\star}, \boldsymbol{S}_{\epsilon \epsilon}^{\star}\right]=\operatorname{argmax}\left[\boldsymbol{S}_{a a}, \boldsymbol{S}_{n n}, \boldsymbol{S}_{\epsilon \epsilon} \mid \hat{\boldsymbol{S}}_{y y}\right],
$$

where $[x \mid y]$ stands for the conditional Probability Density Function (PDF) of $x$ given $y$. From the Bayes' rule, the unknown posterior distribution in Eq. (4) can be written from two known quantities, the likelihood and the prior function, which are detailed hereafter for each contribution of the measured pressure field.

\section{$2.2 \quad$ Acoustic field}

Assuming that the acoustic contribution $\boldsymbol{a}$ results from the propagation of a limited number $K$ of uncorrelated sources with coefficients $\boldsymbol{s}_{j}$ through a transfer matrix $\boldsymbol{H}$, one has:

$$
\boldsymbol{a}_{j}=\boldsymbol{H} \boldsymbol{s}_{j} .
$$

Since in the context of acoustic imaging, the sources are generally unknown and the propagation might be uncertain, the acoustic contribution on the sensors can be more generally written as a linear combination of $\kappa$ latent variables,

$$
\boldsymbol{a}_{j}=\boldsymbol{L} \boldsymbol{c}_{j}
$$

where $\boldsymbol{c}_{j}$ is a vector of $\kappa$ Fourier coefficients, with $K \leq$ $\kappa \leq M$, and $\boldsymbol{L}$ is an $M \times \kappa$ complex matrix that mixes 
these coefficients. The quantities $\boldsymbol{L}$ and $\boldsymbol{c}$ are both unknown, and are not related to any physical quantity. In contrast to a principal component decomposition, the columns of $\boldsymbol{L}$ are not necessarily orthogonal. In this decomposition, $\boldsymbol{L}$ and $\boldsymbol{c}$ are not unique and in general, $\boldsymbol{L} \neq \boldsymbol{H}$ and $\boldsymbol{c} \neq \boldsymbol{s}$.

Unlike the equation (5), this decomposition does not require the knowledge of the transfer function $\boldsymbol{H}$ and it allows the decomposition of the acoustic contribution on the microphones into independent components. This decomposition is referred in the literature as a Factor Analysis and is used in many fields to capture the underlying correlation structure in a dataset [4].

According to the Central Limit theorem applied to the Fourier transform, the latent variables are assigned a Gaussian distribution, and they are also supposed to be a priori independent and heteroscedastic, i.e. with their own variance, which reads

$$
[\boldsymbol{c}]=\mathcal{N}_{\mathbb{C}}\left(\mathbf{0},\left\lceil\boldsymbol{\sigma}_{c}^{2}\right\rfloor\right) .
$$

The notation $\mathcal{N}_{\mathbb{C}}(\boldsymbol{\mu}, \boldsymbol{\Omega})$ refers to the multivariate complex normal distribution with mean $\boldsymbol{\mu}$ and covariance matrix $\boldsymbol{\Omega}$, and the notation $\lceil\boldsymbol{v}\rfloor$ refers to a diagonal matrix with the vector $\boldsymbol{v}$ as diagonal elements. Since the variances of the latent factors are also unknown, they are assigned a prior PDF, under the form of an inverse-gamma law:

$$
\left[\boldsymbol{\sigma}_{c}^{2}\right]=\mathcal{I} \mathcal{G}\left(\boldsymbol{a}_{c}, \boldsymbol{b}_{c}\right) .
$$

The inverse-gamma law is chosen for its positive support and for algebraic convenience - it gives a closed-form expression for the posterior distribution -, see Ref. 5 (p. 42-43) for more details. This choice should not have much impact on the inference problem since, for the further applications (see Secs. 4 and 5), this prior is set "vague" (i.e. with very large variance ; see Sec. A for the values of the prior parameters). Conversely, the choice for heteroscedasticity of the factors is significant and it is motivated by the fact that it is prompt to provide a sparse solution, as shown in Ref. 6. It is equivalent to a mixture of Gaussians with different variances following inverse Gamma laws, which is known to generate a Student-t marginal distribution for the factors $c$ (see Ref. 7, p.102-103).

As no physical interpretation can be made about the mixing matrix $\boldsymbol{L}$, it is assigned, for simplicity, a centered complex multivariate Gaussian, with a normalized variance:

$$
[\boldsymbol{L}]=\mathcal{N}_{\mathbb{C}}\left(\mathbf{0}, \frac{\boldsymbol{I}_{\kappa M}}{\kappa}\right),
$$

with $\boldsymbol{I}_{N}$ the notation for an identity matrix of dimension $N \times N$.

\subsection{Uncorrelated random noise}

In Eq. (1), the vector $\boldsymbol{\epsilon}$ models the sources of microphone self-noise, which can be induced by the measurement setup, the environment, etc. This noise is modeled as totally uncorrelated over the microphones and heteroscedastic. It is statistically described by a diagonal CSM:

$$
\boldsymbol{S}_{\epsilon \epsilon}=\left\lceil\boldsymbol{\sigma}_{\epsilon}^{2}\right\rfloor .
$$

This noise is further supposed to be Gaussian, still according to the Central Limit Theorem applied to Fourier coefficients,

$$
[\boldsymbol{\epsilon}]=\mathcal{N}_{\mathbb{C}}\left(\mathbf{0},\left\lceil\boldsymbol{\sigma}_{\epsilon}^{2}\right\rfloor\right),
$$

and an inverse-gamma distribution is chosen for the variance prior:

$$
\left[\boldsymbol{\sigma}_{\epsilon}^{2}\right]=\mathcal{I} \mathcal{G}\left(\boldsymbol{a}_{\epsilon}, \boldsymbol{b}_{\epsilon}\right) .
$$

The variance of the self-noise is allowed to vary in amplitude from one microphone to another.

\subsection{TBL pressure field}

In the present separation model, the TBL is supposed to develop along a motion-less and rigid wall, and is induced by an incompressible flow parallel to this wall. The TBL is supposed to produce a stationary and homogeneous pressure field over the microphone array, modeled as

$$
\boldsymbol{n}_{j}=p \boldsymbol{\nu}_{j}
$$

where $p$ is a complex scalar amplitude and $\boldsymbol{\nu}$ is a complex random vector that stands for the normalized TBL pressure field. The amplitude term $p$ is supposed to be Gaussian

$$
[p]=\mathcal{N}_{\mathbb{C}}\left(\mu_{p}, \sigma_{p}^{2}\right) .
$$

The vector $\boldsymbol{\nu}$ is modeled as centered Gaussian, with a covariance that is chosen based on a physical TBL covariance model, which reads

$$
[\boldsymbol{\nu}]=\mathcal{N}_{\mathbb{C}}\left(\mathbf{0}, \boldsymbol{\Sigma}_{\nu}^{2}(\boldsymbol{\theta})\right),
$$

where $\boldsymbol{\Sigma}_{\nu}^{2}$ follows an empirical TBL model, which depends on several physical parameters stored in the vector $\boldsymbol{\theta}$. Therefore, the estimated CSM for the TBL contribution is

$$
\hat{\boldsymbol{S}}_{n n}=p^{2} \hat{\boldsymbol{S}}_{\nu \nu}
$$

Any TBL model could be chosen for the TBL prior covariance $\boldsymbol{\Sigma}_{\nu}^{2}$. The one chosen for the present work is that proposed by Corcos [8], which is

$$
\boldsymbol{\Sigma}_{\nu_{k l}}^{2}(\boldsymbol{\theta})=\mathrm{e}^{-\frac{2 \pi f}{U_{c}}\left(\alpha_{x}\left|\boldsymbol{x}_{k}-\boldsymbol{x}_{l}\right|+\alpha_{y}\left|\boldsymbol{y}_{k}-\boldsymbol{y}_{l}\right|-\imath\left(\boldsymbol{x}_{k}-\boldsymbol{x}_{l}\right)\right)},
$$

for a pair of microphones $(k, l)$ with coordinates $\left(\boldsymbol{x}_{l}, \boldsymbol{y}_{l}\right)$ and $\left(\boldsymbol{x}_{k}, \boldsymbol{y}_{k}\right)$, at a given frequency $f$, and $\imath$ being 
the imaginary unit. The flow is along the $x$ direction, and the physical parameters of the flow are $\boldsymbol{\theta}=\left\{U_{c}, \alpha_{x}, \alpha_{y}\right\}$, which are respectively the convection speed of the eddies in the boundary layer, the longitudinal and transverse decay rates of the coherence.

This model has been chosen for several reasons:

- it is written in the space domain, which is necessary since the acoustic contribution is also described in space,

- it has a low number of parameters to be inferred or experimentally estimated,

- as the separation at each frequency line is considered as a separate problem, it is possible to vary the parameter with the frequency to provide a more precise model.

Concerning the last point, the classical Corcos' model assumes that the parameters $U_{c}, \alpha_{x}$ and $\alpha_{y}$ are constant with the frequency, but this does not fully agree with the measurements $[9,10,11]$. Therefore, in the following, a different parameter set $\boldsymbol{\theta}$ is considered for each frequency.

Note that in this probabilistic formulation, the Corcos' model is only a prior information for $\boldsymbol{S}_{n n}$ and does not fully determine the solution of the separation problem. The Bayes estimate of the unknown CSM $\boldsymbol{S}_{n n}$ is given by the maximum of the full posterior distribution, which is proportional to the prior times the likelihood. Therefore, the solution is both driven by the prior and by the data, which means that the inferred TBL CSM is expected to have a correlation structure that derives from a Corcos model, corrected to best fit the measurement data.

\subsection{Implementation of the sampler}

In order to estimate the posterior distribution in Eq. (4), a Gibbs sampler is used, which is a robust Monte Carlo Markov Chain algorithm. Its implementation is easy, but it makes use of the expression of the univariate posterior distribution for each parameter of the model. Its principle is to sample iteratively each random variable from its own distribution conditioned on the current values of the other variables. For each parameter, a chain is built from a fixed number $N_{\text {run }}$ of samples. It can be shown that the stationary distribution converges toward the sought joint distribution [5]. The maximum a posteriori parameters are then estimated by the mean value of the posterior distributions, which tend to be stationary, symmetric and unimodal.

The pseudo-code for the proposed separation approach PFA-Corr is given as Supplementary Material 1 . The prior parameters and initial values used for the

\footnotetext{
${ }^{1}$ See supplementary material for the pseudo-code of the separation procedure.
}

two following applications are given in Sec. A. These values are chosen to be rather general and not very informative.

\subsection{Posterior distributions for the Gibbs sampler}

The hierarchical relation between all the unknown parameters of the problem can be summarily represented using a graph (see Ref. 7, chap. 8). The variables of the model are embodied by nodes, linked together by arrows which indicate their hierarchical relationships. The arrows go from nodes called parents to nodes called children. The parent nodes are variables involved in priors while the child nodes are involved in the likelihood. This graphical representation allows writing the posterior distribution of each parameter $\eta$, by completing the following Bayes formula:

$$
\begin{aligned}
{[\eta \mid \infty] \propto } & \underbrace{[\eta \mid \text { Parents of } \eta]}_{\text {Prior }} \\
& \times \underbrace{\prod_{i}\left[\mathrm{i}^{\text {th }} \text { child of } \eta \mid \text { Parents of } \mathrm{i}^{\text {th }} \text { child }\right]}_{\text {Likelihood }},
\end{aligned}
$$

where " $\mid \infty$ " is to be understood as "conditioned to all the other variables of the model". The graph representing the separation model is given in Fig. 1. From this graph and from Eq. (18), the posterior distribution of each parameters required for the implementation of the Gibbs sampler are given in Supplementary Material $^{2}$. Note that these posteriors are written to account for CSM input data and a block sampling strategy is adopted $[6,12]$ in order to improve to convergence of the sampler.

\section{Estimation of the TBL param- eters}

In order to identify the unknown matrix $\boldsymbol{S}_{n n}$, the covariance given by the Corcos' model in Eqs. (15) and (17) has to be known. Two procedures are proposed to determine the Corcos' parameters $\boldsymbol{\theta}=\left\{\alpha_{x}, \alpha_{y}, U_{c}\right\}$. First, a least squares regression is performed before the denoising and second, a Bayesian inference approach is added within the Bayesian separation procedure.

\subsection{Least squares regression}

A first approach to estimate the Corcos' parameters is inspired from Ref. 11. A Non-Linear Least Squares

\footnotetext{
${ }^{2}$ See supplementary material for the posterior expressions and calculation details.
} 


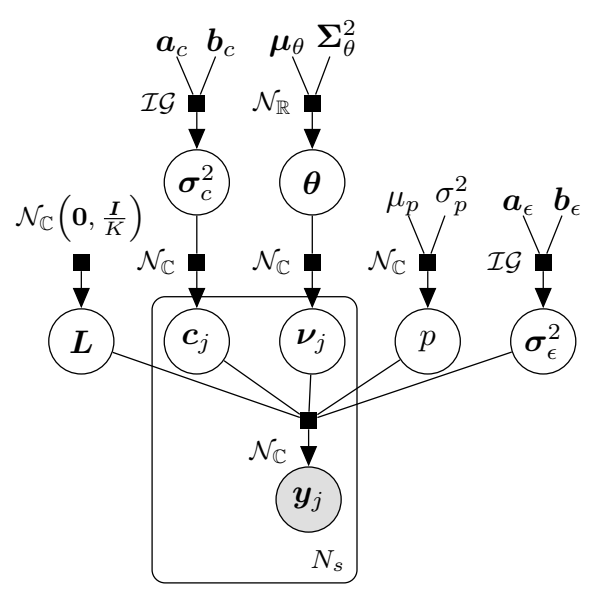

Figure 1: Bayesian hierarchical graph for the separation model of PFA-Corr. The shaded case indicates the observations, the other circles stand for variables to be inferred and the constant hyperparameters are denoted without any border. The dashed box indicates a duplication of the pattern (here for each snapshot).

(NLLS) fitting is performed on the measurement CSM before the separation procedure, which solves the following optimization problem at each frequency:

$\left(U_{c}, \alpha_{x}, \alpha_{y}\right)^{\star}=\operatorname{argmin}\left\|\frac{\hat{\boldsymbol{S}}_{y y}}{\frac{1}{M} \operatorname{tr}\left(\hat{\boldsymbol{S}}_{y y}\right)}-\boldsymbol{G}\left(U_{c}, \alpha_{x}, \alpha_{y}\right)\right\|_{F}^{2}$,

where $\operatorname{tr}(\boldsymbol{A})$ is the trace operator, $\|\boldsymbol{A}\|_{F}$ is the Frobenius norm and each element of the matrix $\boldsymbol{G}$ of indices $k, l=1, \ldots, M$ is given by

$$
\boldsymbol{G}_{k l}\left(U_{c}, \alpha_{x}, \alpha_{y}\right)=\mathrm{e}^{-\frac{2 \pi f}{U_{c}}\left(\alpha_{x}\left|\boldsymbol{x}_{k}-\boldsymbol{x}_{l}\right|+\alpha_{y}\left|\boldsymbol{y}_{k}-\boldsymbol{y}_{l}\right|-\imath\left(\boldsymbol{x}_{k}-\boldsymbol{x}_{l}\right)\right)} .
$$

This method is fast and simple to implement since many ready-to-use functions exist to solve NLLS problems.

However, after this NLLS procedure, the TBL parameters may be biased, since the acoustic part is not taken into account. Alternatively, it is possible to adjust the TBL parameters with Bayesian inference.

\subsection{Bayesian inference}

The TBL parameters can be inferred directly within the Bayesian separation approach, along with all the other unknown parameters of the model.

In this case, they have to be assigned a prior distribution. A possible prior that easily integrates prior information is the multivariate Gaussian:

$$
[\boldsymbol{\theta}]=\mathcal{N}_{\mathbb{R}}\left(\boldsymbol{\mu}_{\theta}, \boldsymbol{\Sigma}_{\theta}^{2}\right)
$$

The prior mean and covariance, $\boldsymbol{\mu}_{\theta}$ and $\boldsymbol{\Sigma}_{\theta}^{2}$, can be known either from physical considerations, or from the NLLS regression introduced before.

As no closed-form of the posterior can be developed for the sampling, a Metropolis-Hastings step is implemented within the Gibbs sampler [13] ${ }^{3}$.

\section{Validation on wind tunnel measurements}

The PFA-Corr method is first tested on measurements performed in a wind tunnel with a closed test section. The acoustic source powers and locations are known and the flow is controlled. In this situation, the validation is done by measuring the acoustic and aerodynamic contributions separately. As the acoustic sources are loudspeakers inside the tunnel roof, their effect on the flow is negligible. Conversely, the flow has a slight impact on the propagation of the acoustic waves, and the subsequent perturbation can be quantified. It remains quite small considering the flow speed therefore, the measurement of the sources with flow is considered as the sum of the two independent contributions.

\subsection{Experimental setup}

The measurements are performed in a wind-tunnel at École Centrale de Lyon (LMFA laboratory), shown in Fig. 2(a). As shown in the sketch in Fig. 2(b), two sources are mounted in the ceiling of the testsection, excited by two uncorrelated white noises. An array composed of 76 Microelectromechanical Systems (MEMS) microphones is mounted in the floor of the section, arranged as shown on Fig. 2(c). The microphone interspacing varies from $0.2 \mathrm{~cm}$ to $27.4 \mathrm{~cm}$. The acquisitions are performed synchronously, during $30 \mathrm{~s}$, and the CSMs are computed with a frequency resolution of $16 \mathrm{~Hz}$, Hann window and $66 \%$ overlapping rate (the apparent number of snapshots is thus $994^{4}$ ).

Three measurements are performed with the MEMS array:

- configuration A : with the sources switched on and without flow,

- configuration T30 : without sources and with a flow at $30 \mathrm{~m} / \mathrm{s}$,

- configuration AT30 : with the sources switched on and the flow at $30 \mathrm{~m} / \mathrm{s}$.

The objective is to separate the acoustic and aerodynamic contributions from the measurements AT30 and

\footnotetext{
${ }^{3}$ See supplementary material at for more details about the Metropolis-Hastings within Gibbs implementation.

${ }^{4}$ The apparent number of snapshots takes into account the window type and the overlapping rate to compensate for the redundancy between the snapshots induced by the overlapping (see for example Refs. 14 and 15).
} 
compare them with the baseline measurements $\mathrm{A}$ and T30. Note that the proposed separation method does not compensate for the convection effect on the acoustic part. Therefore, the identified acoustic part cannot be exactly similar to the not-convected measurement A [3].

\subsection{Wavenumber-frequency maps of the measurements}

The wavenumber-frequency content of the measurements is shown in the form of a $k_{x}-f$ map at $k_{y}=0$ $\mathrm{rad} / \mathrm{m}$ in Fig. 3 ( $f$ being the frequency, $k_{x}$ and $k_{y}$ the wavenumbers longitudinal and transverse to the flow respectively). It is computed by means of a plane wave beamforming process, skipping the autospectra thanks to the following vectorized computation [16]:

$$
A_{i}=\frac{\operatorname{vec}\left(\boldsymbol{w}_{i} \boldsymbol{w}_{i}^{H}\right)^{H} \operatorname{vec}\left(\overline{\boldsymbol{S}}_{y y}\right)}{\left\|\operatorname{vec}\left(\boldsymbol{w}_{i} \boldsymbol{w}_{i}^{H}\right)\right\|_{2}^{2}}
$$

at each frequency, where $\|\cdot\|_{2}$ is the $\ell_{2} \operatorname{norm}, \operatorname{vec}(\boldsymbol{A})$ stands for the vectorization of the matrix $\boldsymbol{A}$ and the notation $\overline{\boldsymbol{A}}$ indicates that the diagonal elements of the matrix $\boldsymbol{A}$ are set to zero. The steering vectors are defined by

$$
\boldsymbol{w}_{i}=\mathrm{e}^{\imath k_{x_{i}} \boldsymbol{x}+\imath k_{y_{i}} \boldsymbol{y}}
$$

where $\boldsymbol{x}$ and $\boldsymbol{y}$ are the coordinates of the microphones along and transverse to the flow respectively.

On these $k_{x}-f$ maps is also plotted the acoustic ellipse - that appears as a cone in the $k_{x}-f$ representation - bounded by $[17,18]$ :

$$
k_{x}=\frac{2 \pi f \cos \theta}{c_{0}+U_{c} \cos \theta} \quad \text { and } \quad k_{y}=\frac{2 \pi f \sin \theta}{c_{0}+U_{c} \cos \theta}
$$

with $0 \leq \theta \leq 2 \pi$ and $c_{0}$ the speed of sound in the ambient air at rest.

These maps show that the two domains overlap below $500 \mathrm{~Hz}$. At low frequencies, the convective ridge is duplicated along the $k_{x}$ axis because of of the grating lobes. This aliasing is also visible above $3 \mathrm{kHz}$, on the map of the configuration A, leading to some duplications of the acoustic spot. It is also clear that the order of magnitude of each contribution is very different depending on the frequency.

Moreover, it appears that the AT30 measurement does not exactly correspond to the sum of the contributions measured in the $\mathrm{A}$ and T30 configurations, especially below $1500 \mathrm{~Hz}$, where the acoustic field is very low, probably due to an effect of the flow on the loudspeakers.

\subsection{Estimation of the TBL parameters}

The TBL parameters estimated using the NLLS regression on the raw AT30 measurements are shown in
Fig. 4, along with the parameters obtained from the Bayesian separation process - using the NLLS values as priors. In this Figure, for a better comparison with the literature, the convection speed is normalized with the flow speed $U_{\infty}$ and the parameters of coherence decay rates are shown in terms of coherence lengths, computed using

$$
L_{c_{x, y}}=\frac{U_{c}}{2 \pi f \alpha_{x, y}} .
$$

The TBL parameters from the Bayesian inference slightly differ from those obtained with NLLS, especially at low frequencies.

As explained in Sec. 2.4, the estimated TBL CSM is not expected to have exactly a Corcos correlation structure. Indeed, the Corcos' model is only used as a prior information and the inferred TBL contribution also depends on the likelihood of the measurements. Therefore, the TBL parameters inferred with PFA-Corr may be difficult to interpret as Corcos' parameters, which can explain the difference obtained at low frequencies where the acoustic contribution is very small. However, this correction is small enough to conclude that in the case where the array allows an efficient regression, it is not necessary to do a Bayesian inference of the TBL parameters, and the NLLS values can be used as is to perform the separation. Especially since it has been verified that the effect of these small differences on the TBL parameters is small in terms of reconstruction error of each contribution [19].

The estimated convection speed follows a classical decrease, as described in the literature [11, 10]. Similarly, the evolution of the coherence lengths with frequency is well known [9]. Above $5 \mathrm{kHz}$, the interspacing of the microphones is too high to provide accurate estimates.

\subsection{Reconstruction of the acoustic au- tospectra}

The diagonal elements of the measurement CSM are the most impacted by the TBL, and thus the reconstruction of the acoustic autospectra is challenging. The reconstructed acoustic autospectra are plotted, as well as their associated error obtained from the baseline measurement of the source only (configuration A), in Figs. 5(a) and 5(b) respectively. The reconstruction error is calculated as follows:

$$
\delta=\frac{\left\|\operatorname{diag}\left(\boldsymbol{S}_{a a}^{A}\right)-\operatorname{diag}\left(\tilde{\boldsymbol{S}}_{a a}\right)\right\|_{2}}{\left\|\operatorname{diag}\left(\boldsymbol{S}_{a a}^{A}\right)\right\|_{2}},
$$

with $\tilde{\boldsymbol{S}}_{a a}$ the reconstructed acoustic CSM and $\boldsymbol{S}_{a a}^{A}$ the baseline source CSM from configuration A. 


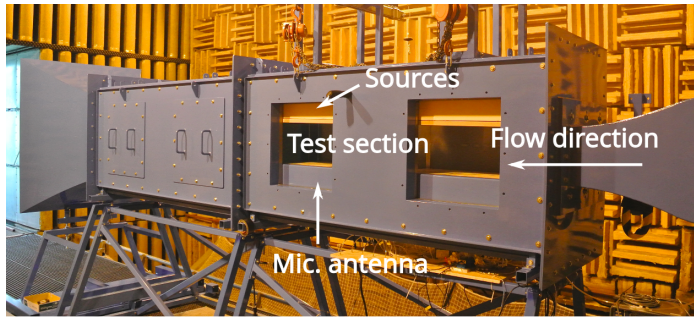

(a) Picture of the facility

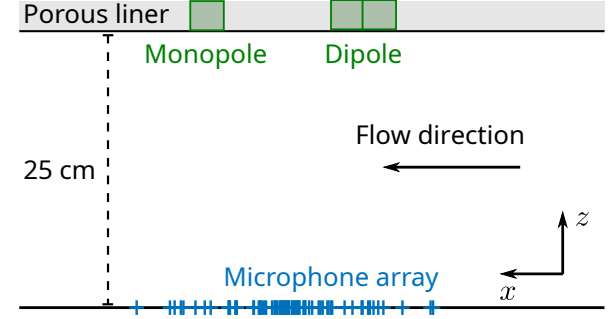

(b) Sketch of the test section (side view)

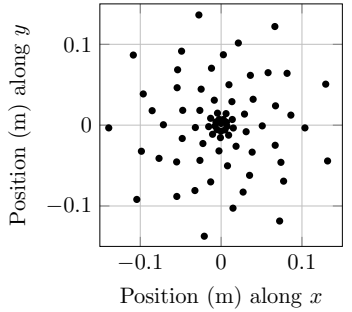

(c) MEMS array design

Figure 2: Description of the experimental setup for the wind-tunnel measurements.

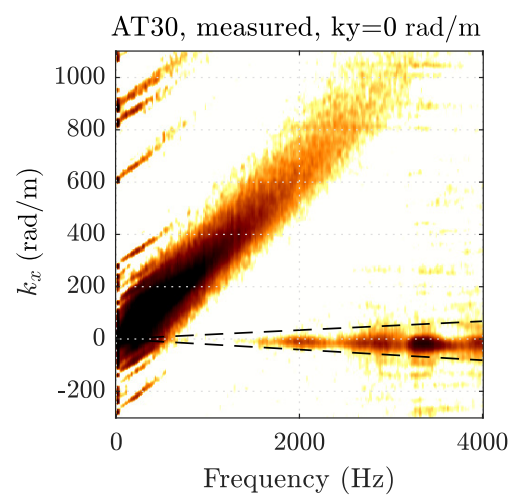

(a) AT30 configuration

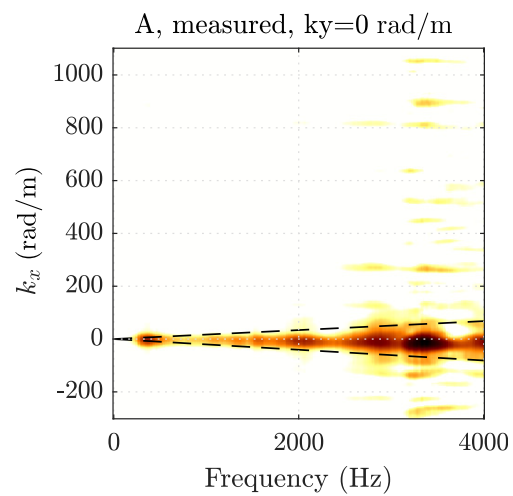

(b) A configuration

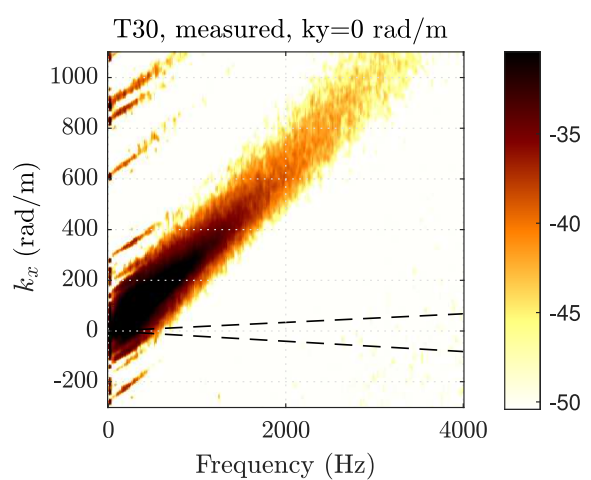

(c) T30 configuration

Figure 3: Wavenumber-frequency maps obtained at $k_{y}=0 \mathrm{rad} / \mathrm{m}$ for the measurements configurations AT30 (a), A (b) and T30 (c). The three maps are scaled with the same color bar (in dB). The two black dashed lines indicate the acoustic domain.

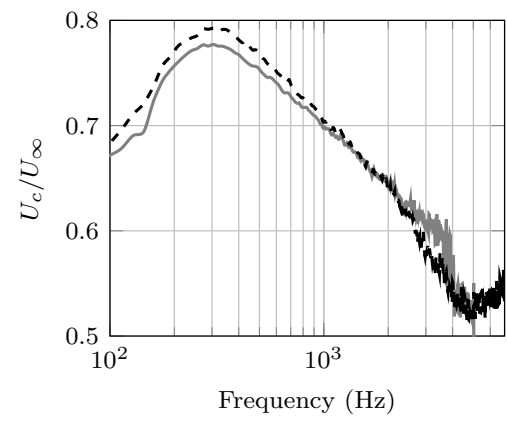

(a) Normalized convection speed

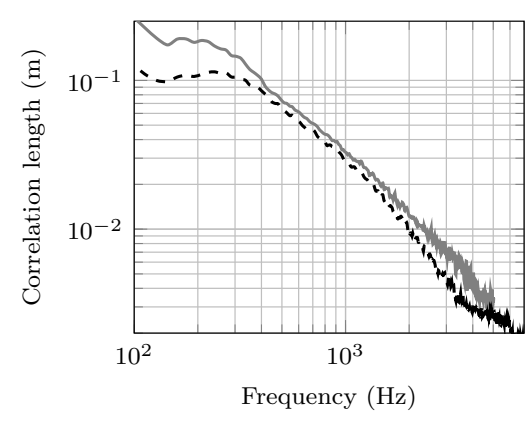

(b) Longitudinal coherence length

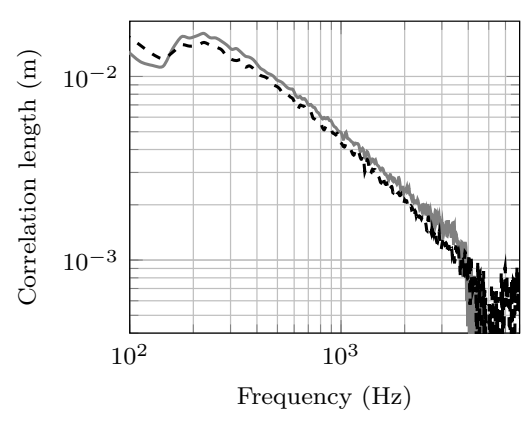

(c) Transverse coherence length

Figure 4: TBL parameters estimated from the NLLS regression on the AT30 measurements (- - - ) and inferred within the PFA-Corr approach (-). 
On these Figures the results from two separation approaches are compared:

- the PFA-Corr approach as given in Sec. 2, based on a correlated TBL prior

- the approach as given in Sec. 2, but based on fully uncorrelated TBL model, i.e. setting $p=0$ in Eq. (13). This model then becomes equivalent to the PFA method [3].

For a comparison of PFA with some methods from the literature, the reader can refer to Ref 3 , where PFA is shown to outperform the other methods on most of the frequency band, on a similar experimental data set.

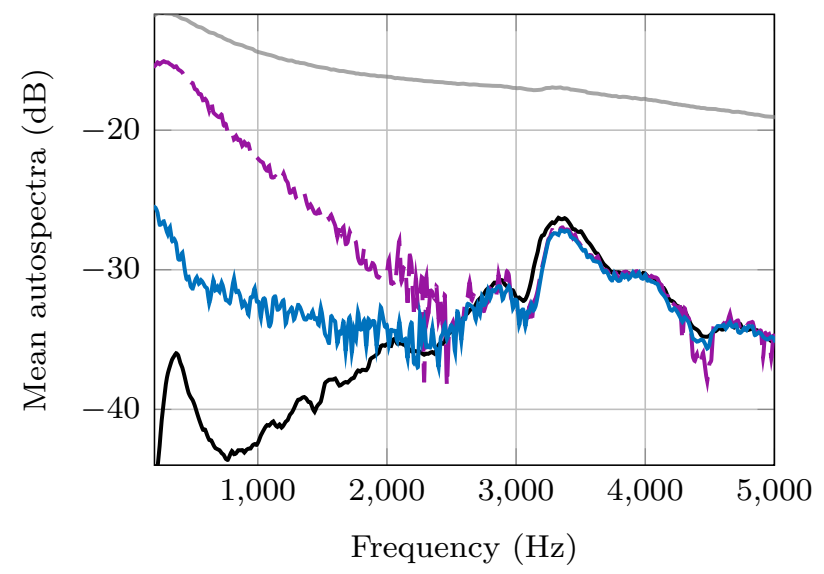

(a) Acoustic autospectra averaged over the microphones

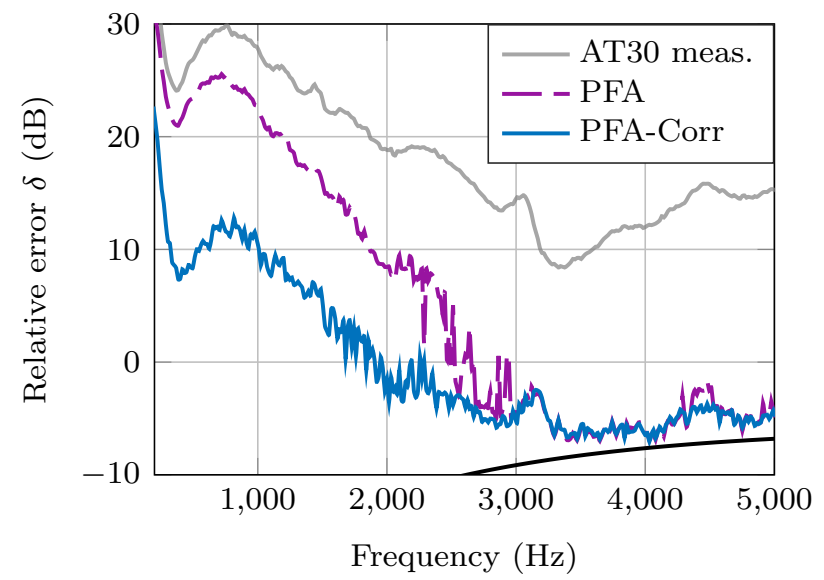

(b) Error on the autospectra based on the T30 measurements

Figure 5: Mean acoustic autospectra (a) and associated error (b) of the raw data (-), of the PFA separation (uncorrelated TBL model) ( - .) and of the PFA-Corr separation (correlated TBL model) (-). On (a) is also plotted the baseline mean autospectrum (-) and on (b) the error due to the convection effect (-) (see Ref. 3 for details on how this effect is evaluated).

The main differences between the two approaches appears below $2.5 \mathrm{kHz}$, which corresponds to the fre- quency range for which the acoustic-to-TBL ratio is very low (as visible in Fig. 3) and where the TBL is highly correlated over the microphones. Therefore, in this low frequency range, the PFA-Corr approach offers a better reduction of the TBL noise on the acoustic autospectra, and also a much lower reconstruction error. Above $2.5 \mathrm{kHz}$, the coherence length in the TBL becomes much lower than the microphone interspacing, and the two approaches thus become equivalent and give very similar results in terms of spectrum level and error.

\subsection{Wavenumber-frequency analysis of the separation results}

In order to complement the previous analyses, the wavenumber-frequency maps of the contributions reconstructed by PFA-Corr are given in Fig. 6. As the autospectra are not taken into account for the calculation of these maps, the dynamic is improved and only the reconstruction of the cross-spectra are represented. Since PFA relies on a diagonal TBL CSM assumption, the wavenumber-frequency maps of its reconstructed contributions are not shown.

For PFA-Corr separation, the $k_{x}-f$ map of the residuals is also given. The residuals are calculated as follows:

$$
\boldsymbol{R}=\hat{\boldsymbol{S}}_{y y}-\left(\tilde{\boldsymbol{S}}_{a a}+\tilde{\boldsymbol{S}}_{n n}+\tilde{\boldsymbol{S}}_{\epsilon \epsilon}\right) .
$$

On the $k_{x}-f$ maps of the acoustic part identified by PFA-Corr (Fig. 6(a)), the convective ridge is well reduced as compared to the measurement map, and even seems totally removed, except below $220 \mathrm{~Hz}$, where the TBL noise is highly correlated over the microphones and where the acoustic sources emit at very low amplitudes. Up to $1500 \mathrm{~Hz}$, the acoustic contribution seems to be not well reconstructed compared to the baseline measurement (Fig. 3(b)), which may be due to the effect of the flow on the loudspeaker, and which could be confirmed by advanced acoustic imaging (in space).

However, the acoustic contribution appears to be removed from the PFA-Corr TBL maps in Fig. 6(b). On the residual map for the PFA-Corr method (Fig. 6(c)), a small part of the convective ridge persists, as well as a small part of the acoustic contribution. However, the residuals from PFA-Corr are rather small, which proves a good agreement between the model and the measurements.

\section{Application to inflight mea- surements}

Another set of data is now employed to test the separation approach. These data are obtained from mi- 


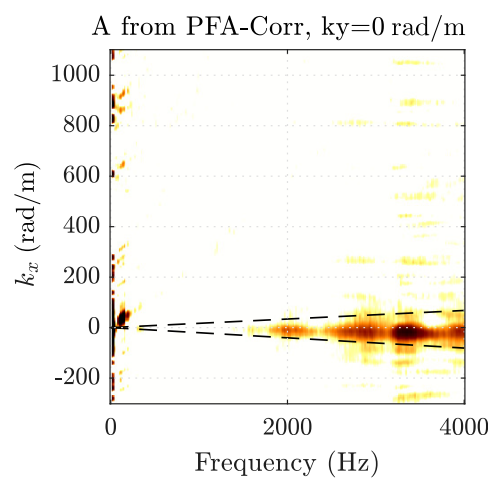

(a) Acoustic part

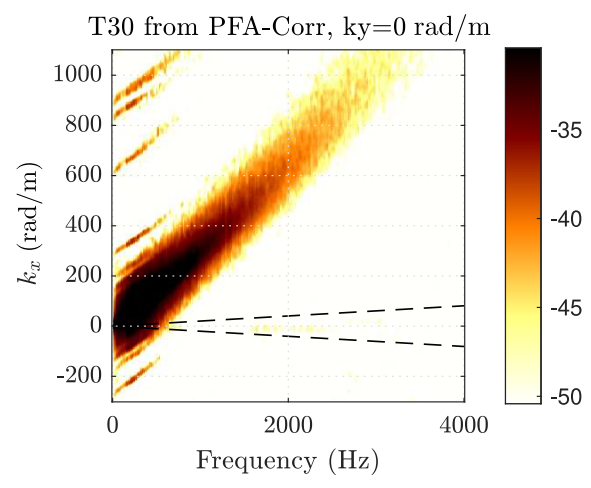

(b) TBL part

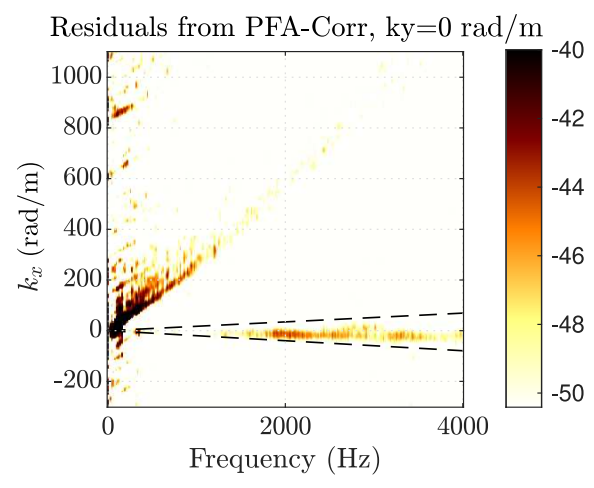

(c) Residuals

Figure 6: Wavenumber-frequency maps obtained at $k_{y}=0 \mathrm{rad} / \mathrm{m}$ from the PFA-Corr separation approach. Identification of the acoustic part (a), the TBL contribution (b) and the residual map (c). Maps on (a) and (b) are scaled with the same color bar (in $\mathrm{dB}$ ) and the residual map is scaled on a smaller dynamic range. The two black dashed lines indicate the acoustic domain.

crophones mounted on the rear fuselage of an inflight aircraft. For this application, the acoustic sources are unknown, even if a strong contribution of the engine jet is expected, and the precise nature of the TBL is also unknown, given that the fuselage is not the flat infinite plate of the Corcos' model.

\subsection{Experimental setup}

The measurements are performed synchronously by 25 microphones, flush mounted on the left side of the fuselage of a large Airbus aircraft. As shown in Fig 7, the array behind the wing, specially designed to characterize the engine jet noise, consists of 5 lines of microphones oriented at 60 degrees from the mean aircraft length axis. The distance between two consecutive lines is about $0.6 \mathrm{~m}$. Each record lasts $60 \mathrm{~s}$, and the CSMs are computed with a resolution of $4 \mathrm{~Hz}$, with $66 \%$ overlapping rate (the apparent number is thus 497). More details on this experimental setup are given in Ref. 20

The measurements are performed for two configurations. The first one is at idle engine speed, and is considered to measure mainly the TBL contribution. The second one is a cruise configuration, at high engine power. For these two configurations, the aircraft is traveling at Mach number 0.85 , at a classical cruise flight altitude.

The recording conditions are very different from the wind-tunnel experiment: the number of sensors is much lower, the flow Mach number is much higher and the physical conditions are known to vary along the fuselage [21], which may lead to inhomogeneous TBL pressure fields over the antenna. Moreover, the microphone arrangement is very regular, which is prompt to produce grating lobes on the beamforming maps.
Note that in the following, for simplicity, the curvature of the fuselage is neglected, and the flow is considered to be orientated along the horizontal axis ( $x$ axis), which is a bit different from what has been observed in the literature [18, 22].

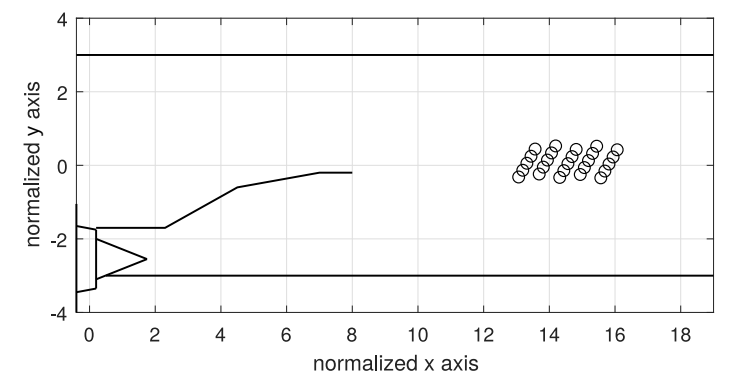

(a) Sketch of the microphone configuration on the fuselage

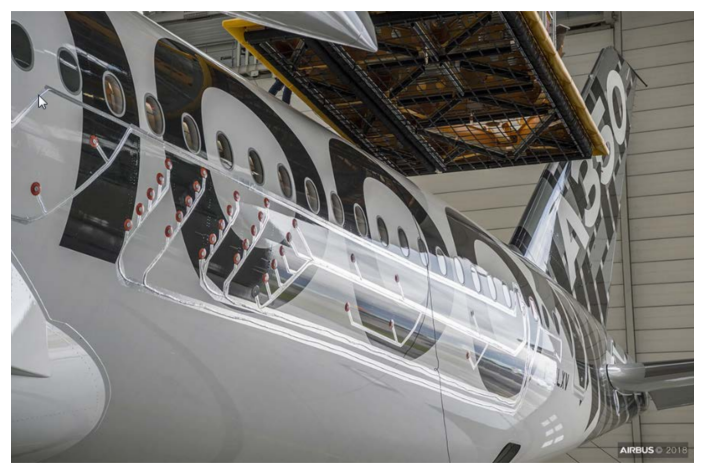

(b) Picture of the microphone antenna, from Ref. 20

Figure 7: Experimental setup for the inflight measurements 


\subsection{Wavenumber maps of the measure- ments}

The wavenumber-frequency maps of the cruise and idle configurations are given in Fig. 8(a) and 8(b). On these maps is also plotted the convected acoustic circle (dashed line), which is strongly shifted toward the negative wavenumbers, due to the high convection speed.

The cruise configuration shows a wavenumber content inside the acoustic circle, compared to the idle configuration. A solid black line also indicates an approximated location of the convective ridge. The position of the ridge increases linearly with the frequency, which supposes that the convection speed is constant with the frequency. The convective ridge and the acoustic content are duplicated many times along the $k_{x}$ due to the grating lobes. At some frequencies, some duplicates of the convective ridge are greater than the main lobe, which is due to the fact that the convective area is not maximum at $k_{y}=0 \mathrm{rad} / \mathrm{m}$. Therefore, some duplicates correspond to regions with higher amplitude.

The TBL contribution dominates the cross-spectra mainly up to normalized frequency 1.7 , therefore, in the following, the separation results are shown only in this low frequency range.

\subsection{Estimation of the TBL parameters}

The Corcos' parameters are first estimated using the NLLS procedure. The resulting convection speed and coherence lengths are given in Fig. 9. In this figure is also plotted the parameters estimated by PFA-Corr, with the NLLS values used as priors. As there is a limit to the minimum microphone interspacing, the TBL parameters can be estimated only at low frequencies up to 3.7. This limit corresponds to a convected wavelength that becomes smaller than the distance between the slanted lines of microphones. Moreover, below the normalized frequency 0.5 , the length of the antenna along the $x$ axis becomes lower than the expected coherence length. For this reason, the longitudinal coherence length estimated with NLLS is overestimated.

For both the NLLS and the PFA-Corr approaches, the coherence lengths are also difficult to infer because of the array geometry which does not contain a lot of microphone lines aligned with the flow direction and transverse to it. Also, as the microphones are flush mounted, they may perturb the TBL. All these variables can explain the difference between these coherence lengths and the values found in the literature, such as in Ref. [23] for similar cases.

As expected from the beamforming maps, the estimated convection speeds are nearly constant with the frequency.

\subsection{Reference-based denoising}

Since no baseline acoustic source measurement is available, the separation result from a reference-based method is further shown in order to be compared with the proposed FA-Corr separation.

The noise affecting fuselage microphones is mainly attributed to the contribution of the TBL. In cases where some noise-free reference signals are available, recorded simultaneously with the microphone array signals, it is possible to deduct the TBL contribution to the microphone measurements. Let $\boldsymbol{x}$ and $\boldsymbol{y}$ stand for a set of reference and array microphone signals, respectively. The CSM of the outputs conditioned by references is given by

$$
\boldsymbol{S}_{y y}^{x}=\boldsymbol{S}_{y x} \boldsymbol{S}_{x x}^{-1} \boldsymbol{S}_{x y} .
$$

This formulation is in accordance with Bendat and Piersol's Conditioned Spectral Analysis [24]: autospectra on the diagonal of $\boldsymbol{S}_{y y}^{x}$ are corresponding to multiple coherent output spectra, the multiple coherence being directly given by the ratio between diagonal terms of $\boldsymbol{S}_{y y}^{x}$ and $\boldsymbol{S}_{y y}$.

Note that this denoising approach assumes that the noise affecting the output signals is not correlated with the references. Even if the assumptions and required data are different for the reference-based denoising and the Bayesian separation approaches, a good agreement between their results will be considered in the following as a cross-validation of both approaches.

For the considered experimental application, reference signals are 6 accelerometers and 9 microphones positioned on the inner side of the fuselage and inside the aircraft cabin, respectively. Those sensors are used as references because they are supposed to be much less affected by the TBL than external microphones since the fuselage acts as a low-pass filter in the wavenumber domain. Moreover, the remaining TBL contribution on the references results from the TBL excitation on the whole fuselage, that is expected to be almost incoherent with the TBL contribution to the 25 microphones covering a very small part of the whole fuselage. Note that interior microphones are affected by interior noise sources, mainly the air conditioning system, but this noise being independent from the TBL, this should not be an issue.

The wavenumber-frequency map of the acoustic contribution reconstructed using the reference-based method is shown in Fig. 8(c). On this map, the convective ridge is still visible, but much lowered as compared to the cruise configuration map. 


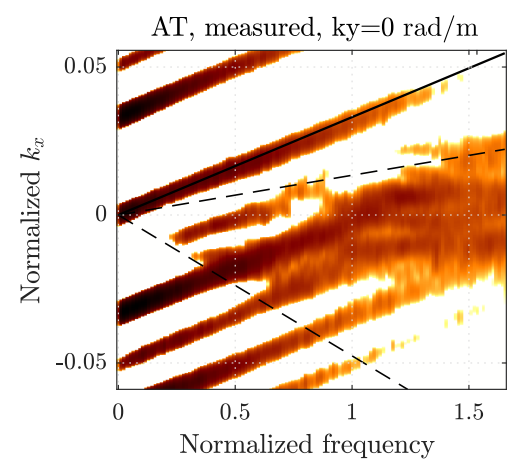

(a) Cruise configuration

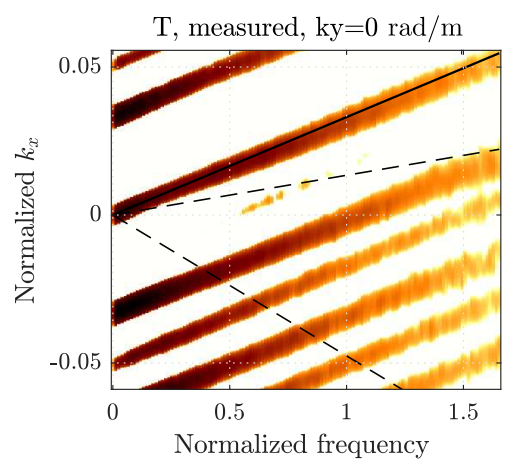

(b) Idle configuration

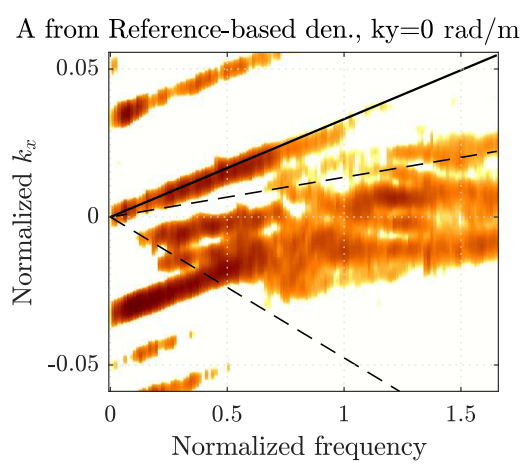

(c) Reference-based denoising

Figure 8: Wavenumber-frequency maps obtained at $k_{y}=0 \mathrm{rad} / \mathrm{m}$ from the measurements at cruise (a) and idle (b) configurations, and from the reference-based separation (c). The three maps are scaled with the same color bar (in $\mathrm{dB}$ ), with $40 \mathrm{~dB}$ of dynamic range. The two black dashed lines indicate the acoustic domain, and the solid line is an approximation of the center of the convective ridge.

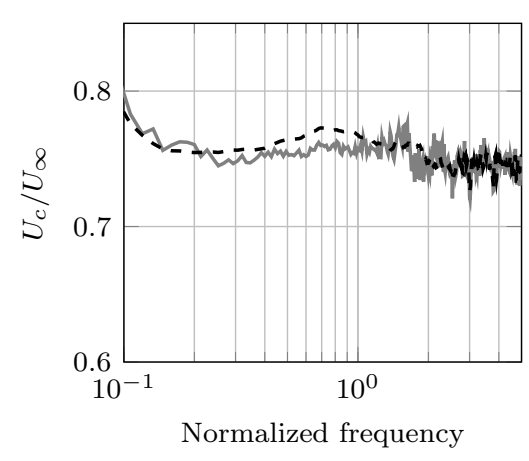

(a) Normalized convection speed

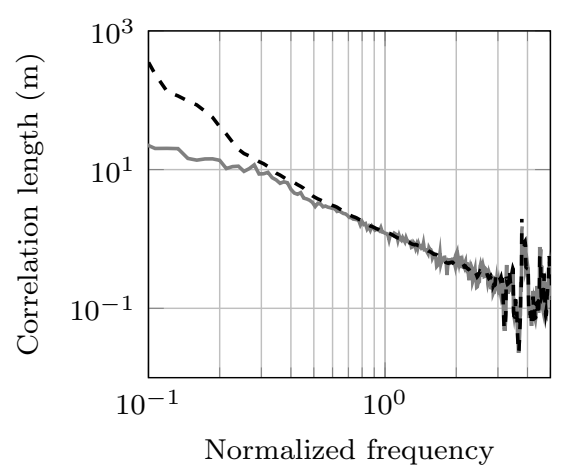

(b) Longitudinal coherence length

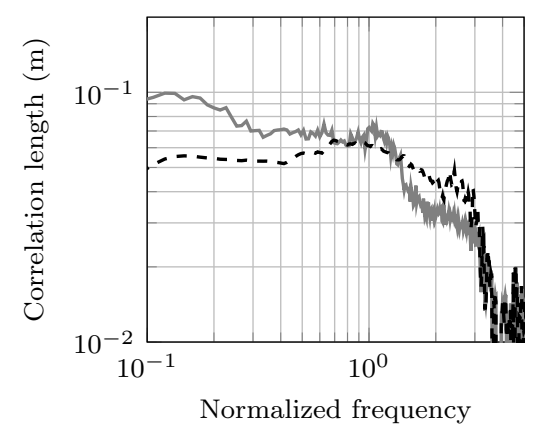

(c) Transverse coherence length

Figure 9: Convection speed (a), longitudinal (b) and transverse coherence lengths (c) estimated using NLLS on the measurement in idle configuration (-- -) and from PFA-Corr (-).

\subsection{Estimation of the acoustic au- tospectra}

In order to have an idea of the reduction of the TBL contribution over the diagonal elements of the acoustic CSMs, the mean autospectra of the acoustic part identified with several approaches are given in Fig. 10. The compared approaches are the reference-based denoising as described in the previous section, PFA and PFA-Corr separations.

The mean autospectra is about $3 \mathrm{~dB}$ higher for the cruise configuration than for the idle configuration. But seeing the level of the various acoustic autospectra, such an augmentation cannot be due to the acoustic contribution. Therefore, this must be due to a modification of the TBL between the two configurations, which is probably not due to the jet since it is rather far from the fuselage (about 6 primary jet nozzle diameters away from the fuselage). Therefore, for the present application, the idle measurement cannot be used to perform a background subtraction such as in Ref. 25)

As for the wind-tunnel application, PFA-Corr offers a greater reduction of the autospectra than PFA, especially in the very low frequency range $(0-1.6)$. On the frequency range 1-4, the measured spectra is dominated by the Broadband Shock-Associated Noise (BB$\mathrm{SAN}$ ), generated by the dual-stream underexpanded supersonic jet [26]. This noise is produced by the interactions of shock cells in the secondary stream with the convected turbulences in the inner and outer shear layers. Harper-Bourne and Fisher[27] propose to model this BBSAN by regularly spaced partially coherent monopoles, which correspond to a low number of acoustic sources, in accordance with the PFA and PFA-Corr assumption. At least one eigenvalue clearly dominates the measurements in the BBSAN frequency 


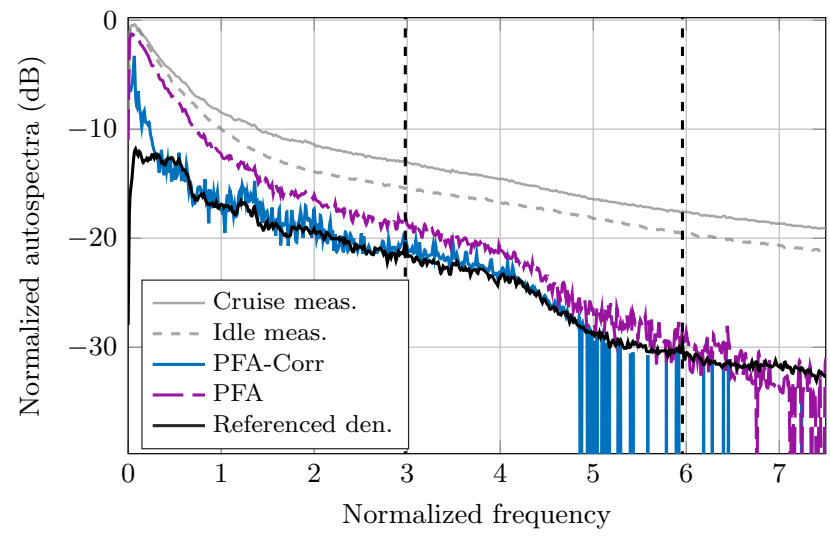

(a)

Figure 10: Acoustic autospectra averaged over the microphones, from the raw measurements $(-)$, background noise measurements $(-=-)$, after referencebased denoising (-), PFA denoising (- -) and PFACorr separation (-). Vertical lines (---) indicate the blade passing frequencies of the engine fan.

range. Whereas at higher frequencies (above normalized frequency 4), no eigenvalue dominates the measurements and both PFA and PFA-Corr fail to reconstruct any coherent spectra. In this frequency range, the reference-based denoising and PFA-Corr are in very good agreement whereas the solution of PFA is higher since its solution is a bit less sparse than the PFACorr's one.

Note that frequency 4 corresponds to the limit above which the half convected acoustic wavelength becomes smaller than the smallest microphone interspacing, and the longitudinal coherence length also becomes smaller than this smallest microphone distance. Therefore, the acoustic part becomes poorly identifiable and the noise tends to be uncorrelated.

In this frequency range, the autospectra denoised by the reference-based denoising also decreases, but it may reach a lower bound given by the number of reference channels used and the residual coherence between the references and the outer microphones.

\subsection{Wavenumber-frequency analysis of the separation results}

The wavenumber-frequency maps of the PFA-Corr separation are given in Fig. 11. The color bar and dynamic range are similar to the measurement maps (Fig. 8). Up to normalized frequency 1 , the reconstruction of the TBL noise is very similar to the measured TBL noise at idle engine speed, which shows that the Corcos prior is suitable for these measurements. Above frequency 1 , the TBL contribution becomes much lower and the identification is less precise.

On the acoustic map, most of the TBL contribution is removed, except in the very low frequencies where a TBL contribution remains at high $k_{y}$ (visible on a $k_{x^{-}} k_{y}$ map) which is then visible on the $k_{x}-f$ map due to the aliasing effect. The acoustic content identified with PFA-Corr has a distribution in the acoustic circle which is similar to the one provided by the referencebased denoising, even if the two methods are based on different requirements and hypotheses. Moreover, the map of the residuals is low. These two observations tend to cross-validate the two approaches.

In Fig. 11(b) and Fig. 8(c), in the acoustic region, two parallel humps increasing linearly on the range $k_{x}=-0.025--0.01 \mathrm{rad} / \mathrm{m}$ and $f=0.25--0.75$ are visible. These acoustic sources are due to the BBSAN and the slope of the humps is related to the convection speed of the turbulences in the primary jet, which is close to acoustic upper limit.

\section{Conclusion}

The PFA-Corr separation problem was conducted within a general framework where no reliable measurement of the TBL contribution is available and where the sources generating the acoustic field are not known (in terms of location, number, type of propagation, etc).

The method described in this paper is a continuation of the PFA method recently developed, which relied on an uncorrelated TBL model, as most of the separation method from the literature. As this assumption is too restrictive at low frequencies, the proposed approach allows to take into account the correlation of the TBL, while requiring very little prior knowledge about the TBL characteristics. While PFA had shown better performance than other state-of-the-art methods, PFA-Corr offers an even better reconstruction of the acoustic autospectra and a better separation of the contributions. This conclusion applies even in presence of aliasing effects, which induce an overlapping of the acoustic and TBL wavenumber content and for which the wavenumber filtering would not be possible to apply.

The versatility of the Bayesian approach makes it possible to extend the model to different needs. For example, for application to flight test measurements, a spatial evolution of the TBL along the fuselage could be taken into account. Other uncertainties can be integrated to the model, such as the angle of attack of the flow on the fuselage. Moreover, other source models could be implemented, such as cyclostationary sources for the study of tonal components of the aircraft engine noise. 


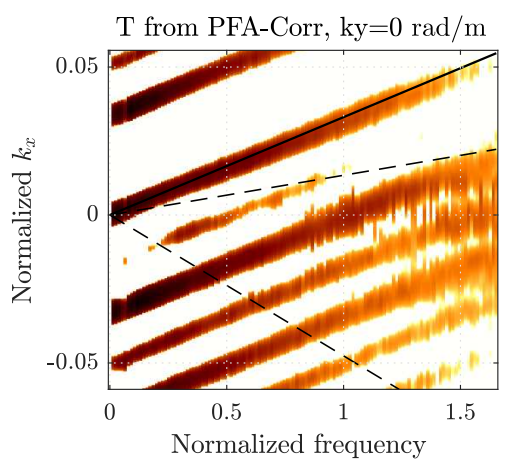

(a) TBL part

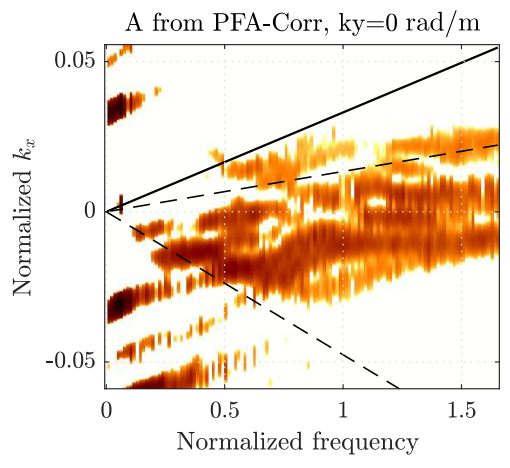

(b) Acoustic part

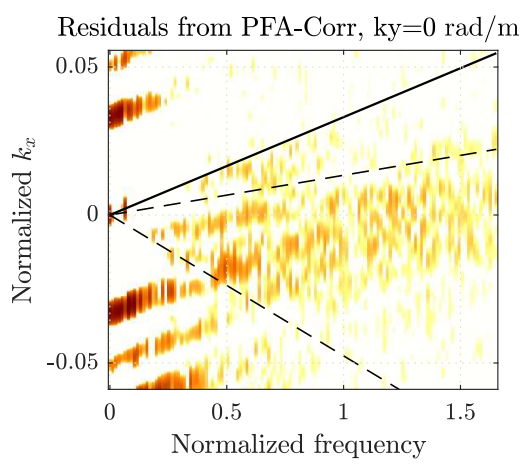

(c) Residuals

Figure 11: Wavenumber-frequency maps obtained at $k_{y}=0 \mathrm{rad} / \mathrm{m}$ from the PFA-Corr separation. Identification of the TBL (a) and the acoustic (b) contributions, and residuals (c). The three maps are scaled with the same color bar as Fig. 8 (in dB), with $40 \mathrm{~dB}$ of dynamic range. The two black dashed lines indicate the acoustic domain, and the solid line is an approximation of the center of the convective ridge.

\section{Acknowledgments}

This work was performed within the framework of the Labex CeLyA of the Université de Lyon, within the program 'Investissements d'Avenir' (ANR-10-LABX0060/ANR-16-IDEX-0005) operated by the French National Research Agency (ANR). This work was also performed in the framework of Clean Sky 2 Joint Undertaking, European Union (EU), Horizon 2020, CS2RIA, ADAPT project, Grant agreement no 754881.

\section{A Initial values and priors}

In hierarchical models such as the one for the PFA-Corr separation, the higher the prior parameter is in the hierarchy, the less influence it will have on the posterior and thus on the separation results. Moreover, Gibbs sampler being guaranteed to converge to the sampled distribution, the separation results are also not supposed to depend on the chosen initial values. However, in order to help the reader to reproduce the presented results, the initial and prior values used in this paper are given in Tab. 1.

In Tab. 1, the eig ${ }_{\kappa}(\boldsymbol{A})$ refers to the $\kappa$ highest eigenvalues of $\boldsymbol{A}$, normalized in order to have $\sum_{\kappa} \operatorname{eig}_{\kappa}(\boldsymbol{A})=$ 1. The matrix $\boldsymbol{S}_{c c}$ does not require to be initialized since it is drawn first. Inverse gamma distributions with scale and shape parameters equal to $10^{-3}$ corresponds to very flat priors, with a small impact on the posterior distribution.

\section{References}

[1] MK Bull. Wall-pressure fluctuations beneath turbulent boundary layers: some reflections on forty years of research. Journal of Sound and vibration, 190(3):299315, 1996.

[2] Edouard Salze, Emmanuel Jondeau, Antonio Pereira, Simon L Prigent, and Christophe Bailly. A new mems microphone array for the wavenumber analysis of wallpressure fluctuations: application to the modal investigation of a ducted low-mach number stage. In 25th AIAA/CEAS Aeroacoustics Conference, page 2574, 2019 .

[3] Alice Dinsenmeyer, Jérôme Antoni, Quentin Leclère, and Antonio Pereira. A probabilistic approach for cross-spectral matrix denoising: Benchmarking with some recent methods. The Journal of the Acoustical Society of America, 147(5):3108-3123, 2020.

[4] David J Bartholomew, Martin Knott, and Irini Moustaki. Latent variable models and factor analysis: A unified approach, volume 904. John Wiley \& Sons, 2011.

[5] Andrew Gelman, John B Carlin, Hal S Stern, David B Dunson, Aki Vehtari, and Donald B Rubin. Bayesian data analysis. Chapman and Hall/CRC, 2014.

[6] Jérôme Antoni, Charles Vanwynsberghe, Thibaut Le Magueresse, Simon Bouley, and Laurent Gilquin. Mapping uncertainties involved in sound source reconstruction with a cross-spectral-matrix-based Gibbs sampler. The Journal of the Acoustical Society of America, 146(6):4947-4961, 2019.

[7] Christopher M Bishop. Pattern recognition and machine learning. Springer, 2006.

[8] G. M. Corcos. Resolution of pressure in turbulence. The Journal of the Acoustical Society of America, 35(2), 1963. 


\begin{tabular}{c|c}
\hline Prior parameters & Initial values \\
\hline $\boldsymbol{a}_{c}=10^{-3}$ & $\kappa=15$ \\
$\boldsymbol{b}_{c}=10^{-3}$ & $\boldsymbol{L}_{0}$ sampled in $\mathcal{N}_{\mathbb{C}}\left(0, \frac{\boldsymbol{I}_{M \kappa}}{\kappa}\right)$ \\
$\boldsymbol{a}_{\epsilon}=10^{-3}$ & $\boldsymbol{\sigma}_{\epsilon_{0}}^{2}=0.5 \operatorname{diag}\left(\hat{\boldsymbol{S}}_{y y}\right)^{\kappa}$ \\
$\boldsymbol{b}_{\epsilon}=10^{-3}$ & $\boldsymbol{\sigma}_{c_{0}}^{2}=\frac{\operatorname{eig}_{\kappa}\left(\hat{\boldsymbol{S}}_{y \boldsymbol{y}}\right)}{2 M} \operatorname{tr}\left(\hat{\boldsymbol{S}}_{y y}\right)$ \\
$\mu_{p}=\sqrt{\operatorname{tr}\left(\hat{\boldsymbol{S}}_{y y}\right) / M}$ & $p_{0}=\mu_{p}$ \\
$\sigma_{p}^{2}=10 \operatorname{tr}\left(\hat{\boldsymbol{S}}_{\boldsymbol{y}}\right) / M$ & $\boldsymbol{\theta}_{0}=\boldsymbol{\mu}_{\theta}$ \\
$\boldsymbol{\mu}_{\theta}$ and $\boldsymbol{\Sigma}_{\theta}^{2}$ from NLLS on $\hat{\boldsymbol{S}}_{y y}$ & $\boldsymbol{S}_{\nu \nu_{0}}=\boldsymbol{\Sigma}_{\nu}\left(\boldsymbol{\theta}_{0}\right)$ (from Eq. (17))
\end{tabular}

Table 1: Prior parameters and initial values for PFA-Corr.

[9] Theodore M Farabee and Mario J Casarella. Spectral features of wall pressure fluctuations beneath turbulent boundary layers. Physics of Fluids A: Fluid Dynamics, 3(10):2410-2420, 1991.

[10] Howard H Schloemer. Effects of pressure gradients on turbulent boundary-layer wall-pressure fluctuations. The Journal of the Acoustical Society of America, 40(5):1254-1254, 1966.

[11] Blandine Arguillat, Denis Ricot, Christophe Bailly, and Gilles Robert. Measured wavenumber: Frequency spectrum associated with acoustic and aerodynamic wall pressure fluctuations. The Journal of the Acoustical Society of America, 128(4):1647-1655, 2010.

[12] D Andrew Brown, Christopher S McMahan, and Stella Watson Self. Sampling strategies for fast updating of gaussian markov random fields. The American Statistician, pages 1-24, 2019.

[13] Steve Brooks, Andrew Gelman, Galin L. Jones, and Xiao-Li Meng. Handbook of Markov Chain Monte Carlo. 2011.

[14] Peter Welch. The use of fast fourier transform for the estimation of power spectra: a method based on time averaging over short, modified periodograms. IEEE Transactions on audio and electroacoustics, 15(2):7073, 1967.

[15] Jérôme Antoni and Johan Schoukens. Optimal settings for measuring frequency response functions with weighted overlapped segment averaging. IEEE transactions on instrumentation and measurement, 58(9):3276-3287, 2009.

[16] Quentin Leclère, Alice Dinsenmeyer, Édouard Salze, and Jérôme Antoni. A comparison between different wall pressure measurement devices for the separation and analysis of TBL and acoustic contributions. In Flinovia-Flow Induced Noise and Vibration Issues and Aspects. Springer, 2021. (In press).

[17] Lars Koop and Klaus Ehrenfried. Microphone-array processing for wind-tunnel measurements with strong background noise. In 14th AIAA/CEAS Aeroacoustics
Conference (29th AIAA Aeroacoustics Conference), page 2907, 2008.

[18] Stefan Haxter and Carsten Spehr. Wavenumber characterization of surfacepressure fluctuations on the fuselage duringcruise flight. In Flinovia-Flow Induced Noise and Vibration Issues and Aspects. Springer, 2019. (In press).

[19] Alice Dinsenmeyer. Probabilistic approach for the separation of the acoustic and aerodynamic wall pressure fluctuations. PhD thesis, Université de Lyon, 2020.

[20] Emmanuel Helffer. From external to internal noise on airbus A350. In 25th International Conference on Sound and Vibrations, July 2018.

[21] Dan Palumbo. Determining correlation and coherence lengths in turbulent boundary layer flight data. Journal of Sound and Vibration, 331(16):3721-3737, 2012.

[22] Stefan Haxter and Carsten Spehr. Comparison of model predictions for coherence length to in-flight measurements at cruise conditions. Journal of Sound and Vibration, 390:86-117, 2017.

[23] Stefan Haxter and Carsten Spehr. Listening to turbulence: Measuring coherence decay at different positions on an aircraft in cruise flight. In 20th $A I A A / C E A S$ Aeroacoustics Conference, page 3064, 2014.

[24] J.S. Bendat and A.G. Piersol. Engineering applications of correlation and spectral analysis. Wiley-Interscience, New York, 1980.

[25] Christopher J Bahr and William C Horne. Subspacebased background subtraction applied to aeroacoustic wind tunnel testing. International Journal of Aeroacoustics, 16(4-5):299-325, 2017.

[26] Christopher KW Tam, Nikolai N Pastouchenko, and Krishna Viswanathan. Broadband shock-cell noise from dual stream jets. Journal of Sound and Vibration, 324(3-5):861-891, 2009.

[27] M Harper-Bourne and M. J. F Fisher. The noise from shock waves in supersonic jets-noise mechanism. In Agard Conference on Noise Mechanisms, number 131, 1973. 